\title{
Gold Nanoparticles as Probes for Nano-Raman Spectroscopy: Preliminary Experimental Results and Modeling
}

\author{
V. Le Nader, ${ }^{1}$ J.-Y. Mevellec, ${ }^{1}$ T. Minea, ${ }^{2}$ and G. Louarn ${ }^{1}$ \\ ${ }^{1}$ Institut des Matériaux Jean Rouxel (IMN)-UMR 6502, 2 rue de la Houssinière, BP 32229, 44322 Nantes Cédex 3, France \\ ${ }^{2}$ Laboratoire de Physique des Gaz et des Plasmas, UMR 8578 CNRS-Université Paris-Sud, Bat. 210, Campus d'Orsay, \\ 91405 Orsay, France
}

Correspondence should be addressed to V. Le Nader, victor.lenader@cnrs-imn.fr

Received 28 July 2011; Revised 14 December 2011; Accepted 29 December 2011

Academic Editor: Ali Passian

Copyright ( $) 2012$ V. Le Nader et al. This is an open access article distributed under the Creative Commons Attribution License, which permits unrestricted use, distribution, and reproduction in any medium, provided the original work is properly cited.

\begin{abstract}
This paper presents an effective Tip-Enhanced Raman Spectrometer (TERS) in backscattering reflection configuration. It combines a tip-probe nanopositioning system with Raman spectroscope. Specific tips were processed by anchoring gold nanoparticles on the apex of tapered optical fibers, prepared by an improved chemical etching method. Hence, it is possible to expose a very small area of the sample $\left(\sim 20 \mathrm{~nm}^{2}\right)$ to the very strong local electromagnetic field generated by the lightning rod effect. This experimental configuration was modelled and optimised using the finite element method, which takes into account electromagnetic effects as well as the plasmon resonance. Finally, TERS measurements on single-wall carbon nanotubes were successfully performed. These results confirm the high Raman scattering enhancement predicted by the modelling, induced by our new nano-Raman device.
\end{abstract}

\section{Introduction}

The Raman effect is a well-known phenomenon of the inelastic diffusion of light, with change in the frequency of the incident radiation passing through materials or molecules. In the spectrum of the scattered light, new bands can be observed, and their energies are characteristic of the chemical nature of the investigated object. Raman spectroscopy is a useful tool, which is widely used in academic and industrial laboratories. This nondestructive spectroscopy provides structural information about various materials and can be exploited to build sensors that operate in different areas such as gas detection or bio- and chemical sensing. Generally, these equipments were built from an optical system that analyzes the diffusion of the light and transfers this information to spectroscopic detectors and finally to the electronic system for data processing. Nevertheless, the weakness of the signal does not allow the investigation of single molecules or individual nanoobjects.

However, in the 70s, it was observed and proved [1-3] that Raman intensity can be strongly enhanced using a specific surface-sensitive technique based on the optical properties of noble metals, such as silver or gold. Nowadays, this phenomenon is well known and commonly called Surface Enhanced Raman Scattering (SERS) effect. This enhancement occurs for molecules adsorbed on rough metal surfaces or on metal nanoparticles. The main Raman enhancement contribution is attributed to the excitation of surface plasmons-polaritons resonances (collective oscillations of the electrons localised near the surface of metals) occurring near metallic nanostructures. The interaction of light with metallic nanoparticles is dominated by strong optical resonances due to the excitation of localized surface plasmons. One of the main consequences of this surface plasmon resonance is the high electromagnetic field created at the surface of the nanoparticle, which significantly enhanced the Raman scattering. The scattering signal can be enhanced by 10 orders of magnitude meaning that this technique may detect single molecules and that the characterization of individual nanoobjects becomes possible [4-7]. This possibility of using colloidal dispersions of silver (Ag) or gold $(\mathrm{Au})$ nanoparticles in aqueous solutions as a method 


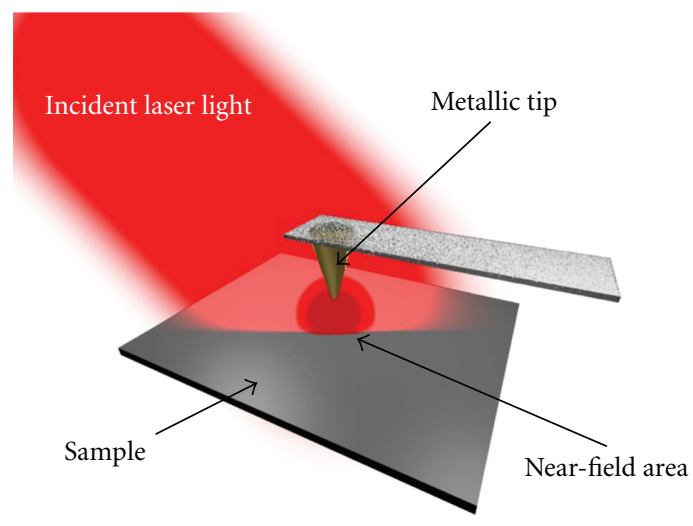

Figure 1: Schematic representation of Tip-Enhanced Raman Spectroscopy in the side-illumination mode.

for enhancing Raman scattering was first demonstrated by Creighton et al. [8]. Note that the intensity of the electromagnetic field strongly depends on the nanoparticle size, shape, and interparticle distance. Such high enhancement factors are also obtained from well-defined nanostructures, like nanoparticles [9-11], nanoholes [12] or nanotips [13]. Hence, Tip-Enhanced Raman Spectroscope (TERS) [14-19] exploits this effect induced by a nanotip, derived from the local probe microscopy techniques, among the Atomic Force Microscopy (AFM) is the most notorious example.

TERS principle consists in the illumination of a sharp metallic tip by a monochromatic light in order to benefit from plasmons resonance and antenna effects. Theoretical studies show that a strongly localized electric field can be created near the tip apex. The strong field is very localized showing strong gradients and thus ensuring both the very good nanometer scale spatial resolution and the enhancement of the optical scattering signal for detection. Finding optimal conditions for achieving a maximal localized field thus becomes one of the most critical issues in tip-enhanced spectroscopies. The field confinement is readily achieved if the polarization vector of the excitation light is orientated along the direction of the tip and if the tip-surface distance is very low. In these conditions, the surface charge density oscillates and very high electromagnetic field amplitudes arise at the tip apex, as a function of the induced-dipole strength. As schematized in Figure 1, a strong confinement and enhancement of the electromagnetic field at the vicinity of the tip apex can occur.

Conventional Raman scattering is known as a very weak light-matter interaction phenomenon, whose cross section is usually $10^{-8}$, much weaker than the cross section of infrared absorption [20]. Additionally, the spatial resolution of Raman spectroscopy is limited by the Rayleigh criterion (theoretical diffraction limit $\sim \lambda / 2$ ). TERS spectroscopy is a way to overcome these limits, and the success of such devices depends on the ability to focus the laser beam on the apex of a sharp metallic probe, located in the nanoscale vicinity ( 1 to 10 nanometers) of a nanoobject or a surface. Since the first experimental published results, worldwide research groups have implemented several TERS configurations. Two types of setups can be distinguished based on light reflection or light transmission.

In the transmission configuration, the scattered light is collected from the opposite side of the sample, which is illuminated by a monochromatic source [21-26]. Generally, the incident beam is focused under the tip apex through the sample. This configuration uses microscope objectives with high Numerical Aperture (NA), including oil-immersion objectives, improving thus the collection efficiency and minimizing the far-field contribution. However, this configuration is limited to optically transparent samples or very thin substrates.

In the backscattering mode, the incident beam does not cross the sample [27-31]. This configuration requires the use of objectives with a numerical aperture ranging from 0.25 to 0.6 . Consequently, in backscattering configuration, the background signal due to the far-field is higher than in transmission mode. This problem can be partially solved by using suitable light polarization $[32,33]$. In spite of this drawback, the backscattering configuration is very interesting due to its versatility. On the contrary to the transmission operation mode, the backscattering one is efficient for the investigation of all types of samples (transparent or not), in particular metallic substrates where plasmonic tip-sample coupling can highly improve the Raman scattering by the local enhancement of the signal.

In this work, we introduce an experimental confocal TERS device in reflection configuration (side illumination) developed in our laboratory and dedicated to nanoobjects investigation. The presented TERS device is based on a commercial Raman spectrometer coupled with a tip-probe nanopositioning system. As plasmon resonance of gold metals occurs in the visible range, special tips improved by gold nanoparticles grafted on the silica tips were designed. Finally, experimental results recorded on single-wall carbon nanotubes (SWCNT) are presented and compared with finite elements analysis.

\section{Experimental Setup}

2.1. Tip-Probe Preparation. The tip accounts as central element in our device. The lateral resolution depends on the tip dimensions (aperture angle, apex diameter). As underlined above, particular optical properties of noble metals can significantly increase Raman scattering. There is a large variety of technical possibilities to elaborate metallic probes and several report efficient probes for TERS spectroscopy [19, 34-37]. The most frequently used are the electrochemical etching of solid metal rods and the thermal evaporation technique to deposit metal on sharp tips.

Here, we describe an alternative method based on the physical anchoring (electrostatic) of gold nanoparticles on a sharp silica tip. In the first step of the process, multimode fibers $(125 \mu \mathrm{m}$ core diameter $)$ are etched using the substitute-sheath etching method [38]. After removing 


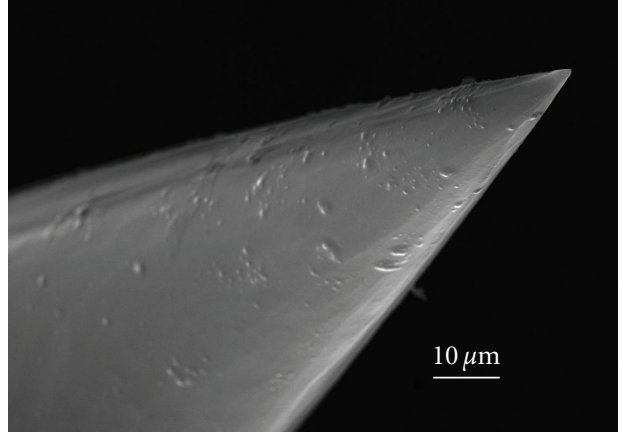

(a)

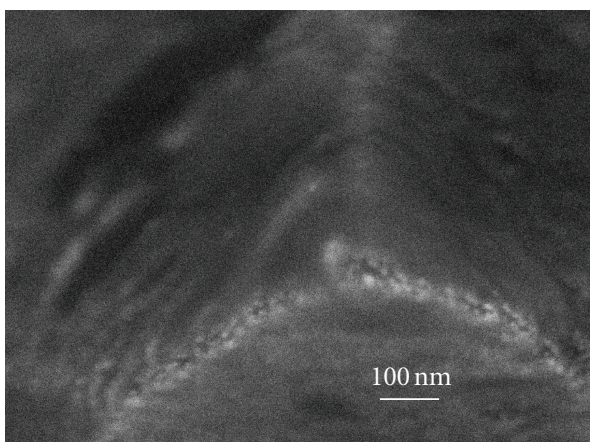

(b)

FIGURE 2: SEM images of tips coated with $15 \mathrm{~nm}$ gold nanoparticles: (a) 1300x magnification. (b) 85000x magnification. At this magnification, nanoparticles on the tip apex can be observed.

the jacket (around $2.5 \mathrm{~cm}$ length from the free extremity of the fiber) by mechanical stripping, a substitution sheath is obtained using a liquid wax. Then optical fibers are immersed for $4 \mathrm{~h}$ in aqueous HF solution at room temperature, leading to the fiber etching following a conical shape. Afterwards the wax coating is simply removed mechanically (Figure 2(a)).

The second step consists of the grafting of gold nanoparticles on the tapered optical fiber. A colloidal solution is obtained from a mixture of chloroauric acid solution $\left(\mathrm{HAuCl}_{4}\right)$ and a trisodium citrate solution $\left(\mathrm{Na}_{3} \mathrm{C}_{6} \mathrm{H}_{5} \mathrm{O}_{7}\right)$ [11]. A Dynamic Light Scattering (DLS) experiment revealed that the diameter of the nanoparticules was about $15 \mathrm{~nm}$. Finally, the Au nanoparticles are immobilized at the apex of the sharp silica tip by way of a chemical agent. The extremities of the optical fibers are immersed in an APTMS (3aminopropyltrimethoxysilane) solution in order to functionalize the silica surface of the tip. The electrostatic interactions between the positives charges of amines functional groups of the APTMS and the negative charges provided by the citrate embedding the nanoparticles are here exploited to graft the gold colloids.

Figure 2 shows a typical nanotip obtained using this process. After the etching step, one can observe that the extremity of the tip is faceted and the tip apex dimensions are around $50 \mathrm{~nm}$. As detailed in Figure 2(b), the face edges of the probe extremity constitute the preferential sites to graft gold nanoparticles. In particular, gold nanoparticles are caught by the tip apex. Sometimes, just one or a small group of gold nanoparticles emerges from the silica tip apex. Therefore, this tip preparation method is very interesting to reduce the probe dimensions.

2.2. Tip-Probe Nanopositioning System. The approach of such prepared tip to the sample is performed by a nanomechanic system providing accuracy higher than $1 \mathrm{~nm}$. The used piezoelectric system (Solver SNOM, NT-MDT) was managed by a controller (NT-MDT, BL022MT). The piezoelectric head can move the tip-probe position in each $X, Y$ and $Z$ direction, over $40 \mu \mathrm{m} \times 40 \mu \mathrm{m} \times 5 \mu \mathrm{m}$. The lateral resolution given by the manufacturer is lower than $20 \mathrm{~nm}$, while the resolution in $Z$ direction is in the order of $0.1 \mathrm{~nm}$, guaranteeing a reliable tip/sample distance. A feedback control loop, based on the amplitude of the tip oscillation, checks and maintains a constant tip/sample distance.

This mode is called "shear-force" because the tip oscillations are forced to be parallel to the surface of the sample [39, 40]. Near the surface, the shear-force damps the oscillations of the tip. From the resulting electric signal, proportional to the strength of the shear-force applied to the tip, the nanopositioning device adjusts the tip position regarding a setpoint value to keep constant the tip-sample distance. In this work, tips were glued on one prong of a quartz tuning fork (Farnell quartz $32.768 \mathrm{kHz}$ ). Figure 3(a) shows the tip stuck (arrows). The tuning fork is excited by the piezodriver with a frequency close to its own resonance.

\subsection{Coupling of the Optical Setup to the Nanopositioning} System. The last point of the nanoRaman concerns the coupling, in time and space, of the tip with the incident laser beam and the Raman spectrometer. The Raman spectrometer used in this work is a commercial T64000 model from HORIBA Jobin-Yvon. The optical detector is a CCD camera cooled by liquid nitrogen. An optical microscope (BH2-UMA) outfitted with a x100 magnification objective (NA: 0.6, WD: $7.6 \mathrm{~mm}$ ) allows to survey the tip and the sample motions. These movements are managed by an $X Y$ table. Experimental results presented in this work were performed using a monochromatic red light $(645 \mathrm{~nm})$ excitation provided by a coherent Krypton ion laser (Figure 4). The incident laser beam was focused in the vicinity of the tip apex by the objective and the scattered signal was collected by the same objective (backscattering configuration). The scattered light passes then through a simple monochromator, with a diffraction grating of 1800 traces $/ \mathrm{mm}$, allowing thus the best compromise between signal intensity and spectral resolution. Let us remember that the orientation of the electric field with respect to the tip axis plays an important role [41-44].

Numerical simulations made by Sun and Shen reveal that the optimal angle between the incident electric field and the axis of a silver tip is in the $30-35^{\circ}$ range, which corresponds to $55^{\circ}$ between direction of the propagating 


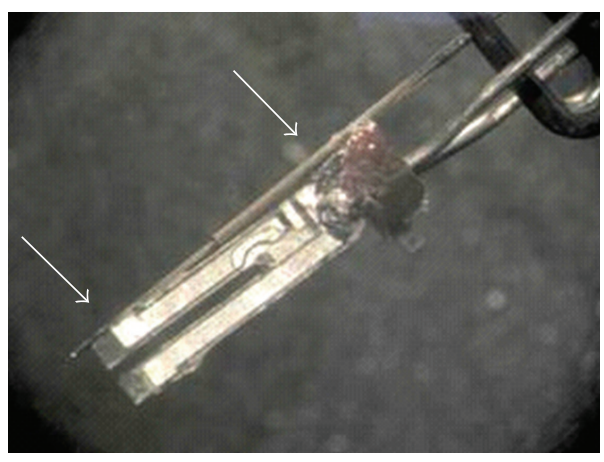

(a)

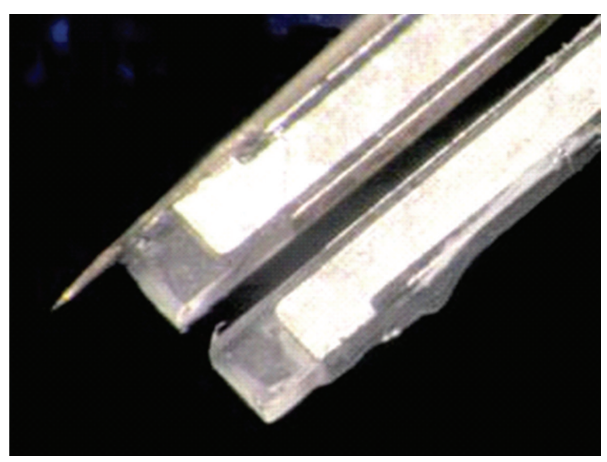

(b)

FIGURE 3: Optical images of the tips glued on a quartz tuning fork. (a) Points of glue are indicated by arrows. (b) Zoom of the quartz fork with the tip.

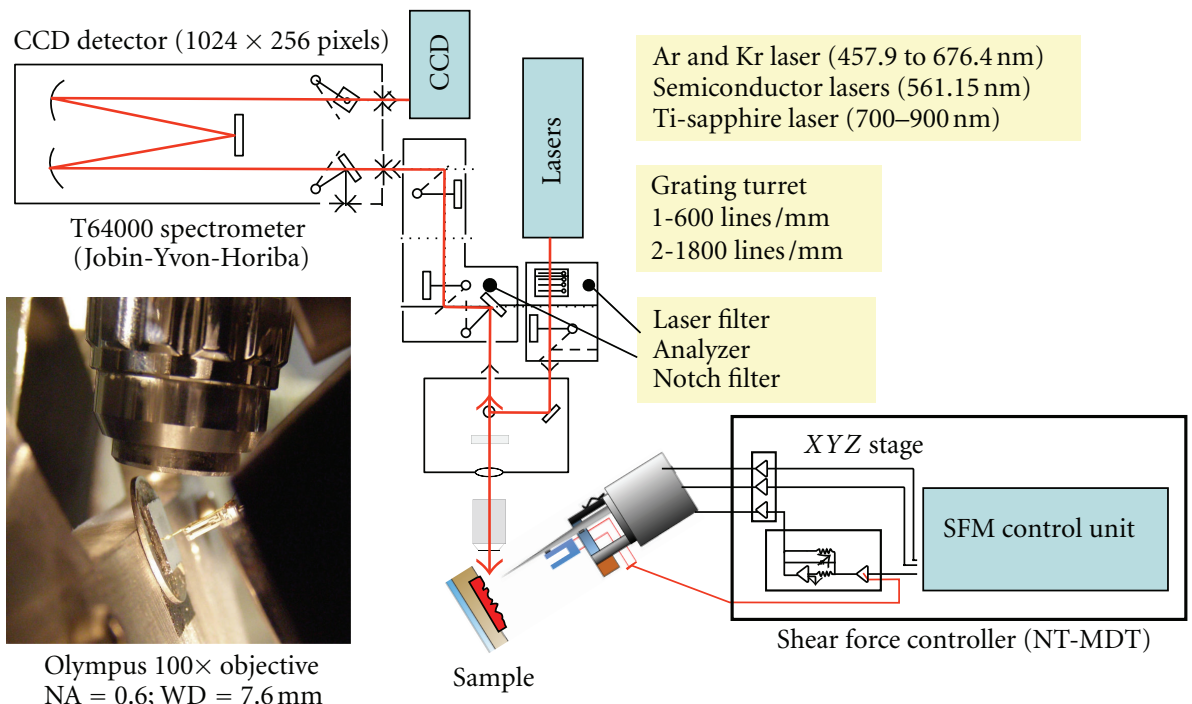

FIGURE 4: A schematic of the tip-enhanced Raman setup with an image of the tip-surface area (inset). The sharp gold tip is scanned through a strongly focused laser beam. The optical signal is detected by combining a spectrometer and a CCD detector. The axis of the illuminating/collecting optics is positioned at $55^{\circ}$ with respect to the tip axis.

wave (p-polarisation) and the tip axis [45]. In agreement with these published results, a specific piezoscanner holder was designed (Inset Figure 4).

2.4. Numerical Modeling. In order to model the experiments, we coupled Finite Element Analysis (FEM) of the electromagnetic field at the vicinity of the surface with the dispersion effects of metals. The equations used to solve the electromagnetic field derive from the Maxwell equations following the simplifying assumptions, particularly about the geometry of the tip. For the resolution of the partial derivative equations (PDEs) and for the space discretization, the software Comsol Multiphysics 3.5 was used [46, 47]. The coupling with the optical properties of gold was implemented under Matlab 7. In this way, the optical effects induced by the plasmon resonance of gold nanoparticles and the partial derivative equations representing the electromagnetic field around the tip were calculated simultaneously as a function of the wavelength taking into account the geometry of the tip.

\section{Results and Discussion}

We designed a spherical gold colloid anchored on the apex of a silica tip close to a smooth golden surface. In this modelling, the angle between the axis of the tip and the direction of the propagating wave was fixed at $55^{\circ}$.

Figure 5(a) shows the enhancement factor $G$, defined as the square of the normalized local electric field amplitude. This map shows the extreme enhancement of the electromagnetic field in a very small area surrounding the tip. In order to determine the wavelength of the incident light which is the most relevant for our system, we calculated the enhancement factor $G$ between the tip and the golden surface as a function of the incident wavelength. Thereby, the FEM calculations predict that the best enhancement is in the range of 550 to 


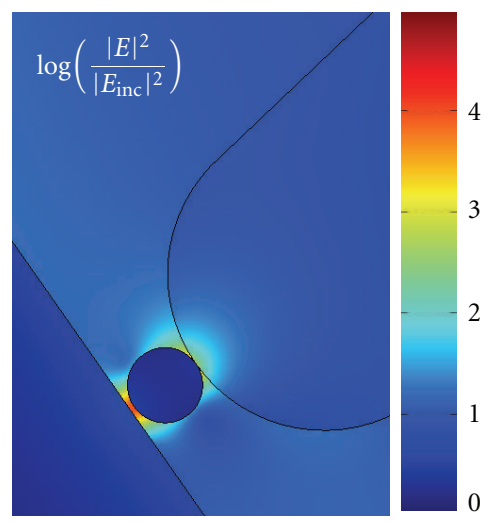

(a)

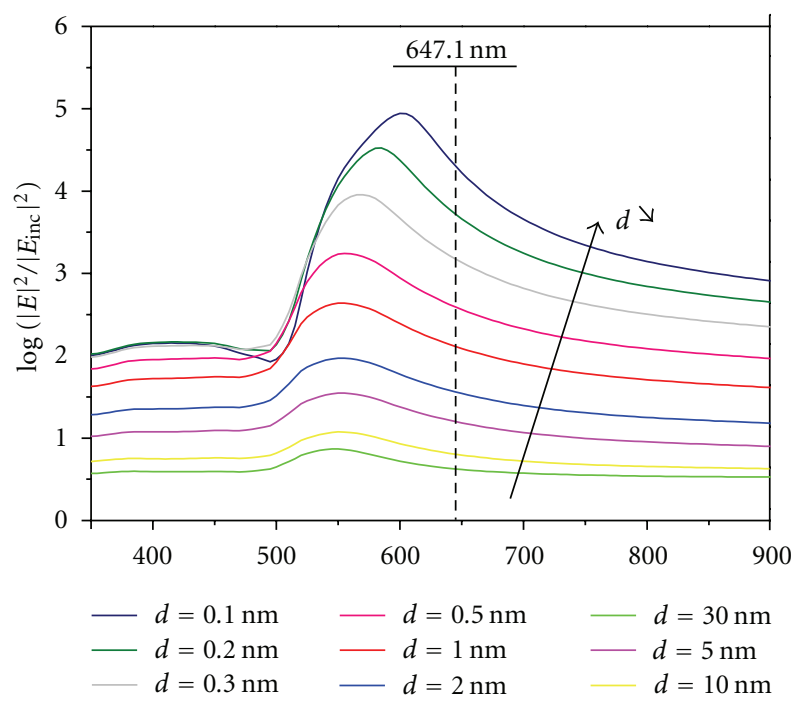

(b)

FIgURE 5: (a) map of the Raman enhancement factor in the vicinity of the surface. For modeling this $2 \mathrm{D}$ structure, only 1 nanoparticle is considered. The diameter of the NPs was fixed to $15 \mathrm{~nm}$, and the distance between tip-surface was taken $1 \mathrm{~nm}$. The wavelength of the Raman excitation was fixed at $650 \mathrm{~nm}$. (b) Finite Elements Analysis (FEA) of the near-field profile of the gold tip under Plasmon resonance excitation, as a function of the wavelength $(400-900 \mathrm{~nm})$ and of the tip-surface distance $(0.1$ to $10 \mathrm{~nm})$.

$650 \mathrm{~nm}$, depending on the distance between the probe and the surface of interest.

In the light of these numerical predictions, we performed TERS experiments on single-wall carbon nanotubes (SWCNT) placed on a smooth gold surface and illuminated by a red monochromatic light (excitation wavelength = $647 \mathrm{~nm})$.

Before discussing in detail the results, let us underline that only carbon nanotubes being in resonance or nearresonance conditions can be detected by Raman spectroscopy. These conditions are reached when the excitation energy corresponds to the energy difference between two Van Hove electronic states. By considering the G-bands profile of the spectrum, presented in Figure 6, and the excitation

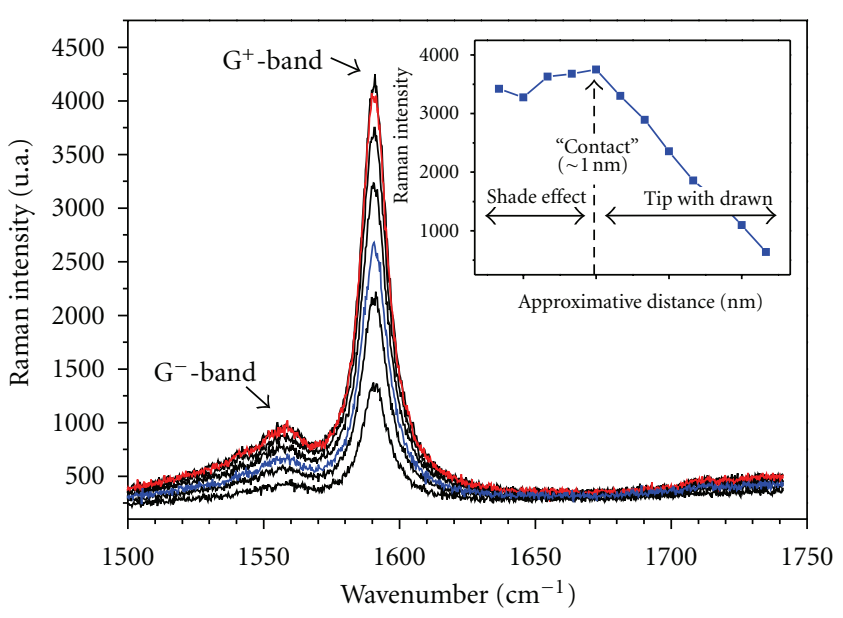

FIGURE 6: TERS signal recorded on an individual SWCNT placed on the surface. Raman spectra are recorded for different tip-SWCNT distance (excitation line: $647.1 \mathrm{~nm}$; laser power: $10 \mathrm{~mW} / \mu \mathrm{m}^{2}$ exposure time: 30 seconds). The inset indicate the intensity of the experimental Raman as a function of the estimated tip-SWCNT distance.

wavelength, we assume that metallic carbon nanotubes are mainly detected. The $\mathrm{G}^{+}\left(1586 \mathrm{~cm}^{-1}\right)$ band profile is Lorentzian. It is accompanied by the $\mathrm{G}^{-}$band at $1566 \mathrm{~cm}^{-1}$ and the D band at $1320 \mathrm{~cm}^{-1}$ and RBM (not shown). The dispersive (D) band indicates the presence of defects such as undesirable $\mathrm{sp}^{3}$ carbon including disordered carbon. Information about nanotubes diameter can be obtained from the radial breathing mode (RBM) at low wavenumbers (usually between $120 \mathrm{~cm}^{-1}$ and $350 \mathrm{~cm}^{-1}$ ).

We focus here on the G-bands, which are attributed to atomic displacements along the tube axis $\left(\mathrm{G}^{-}\right.$band) and atomic vibrations along the circumferential direction $\left(\mathrm{G}^{+}\right.$ band) [48-52]. To quantify the experimental enhancement effect under the tip, Raman spectra were recorded by varying the distance between the nanoprobe and the SWCNT (Figure 6).

One can distinguish two phases during the approach of the tip. During the first phase, the gold nanotip is first roughly approached to the sample by means of a micrometer screw, then during the second phase, a finer approach with the piezoscanner is performed. According to the nanopositioning system, we estimated that the tip-sample distance can vary in the range of $2 \mu \mathrm{m}$ to $2 \mathrm{~nm}$. The spectra show the enhancement of the Raman signal $\left(\mathrm{G}^{+}\right.$-band). By reducing the sample-tip distance from around $2 \mu \mathrm{m}$ to $2 \mathrm{~nm}$, the Raman intensity is 6 times enhanced (contrast). In order to estimate the enhancement factor yielding under the tip, the far-field signal must be separated from the near-field signal. We estimated the average diameter of the focus incident beam at $1.5 \mu \mathrm{m}$, which is generally assumed for the used objective $(\times 100$, NA: 0.6, WD: $7.6 \mathrm{~mm})$. From the modelling, it is possible to assess the area that contains the largest nearfield contribution. Hence, for a diameter of $20 \mathrm{~nm}$ the best signal is expected for a tip-sample distance of $2 \mathrm{~nm}$ and an excitation wavelength of $645 \mathrm{~nm}$. The equation (1) gives us 


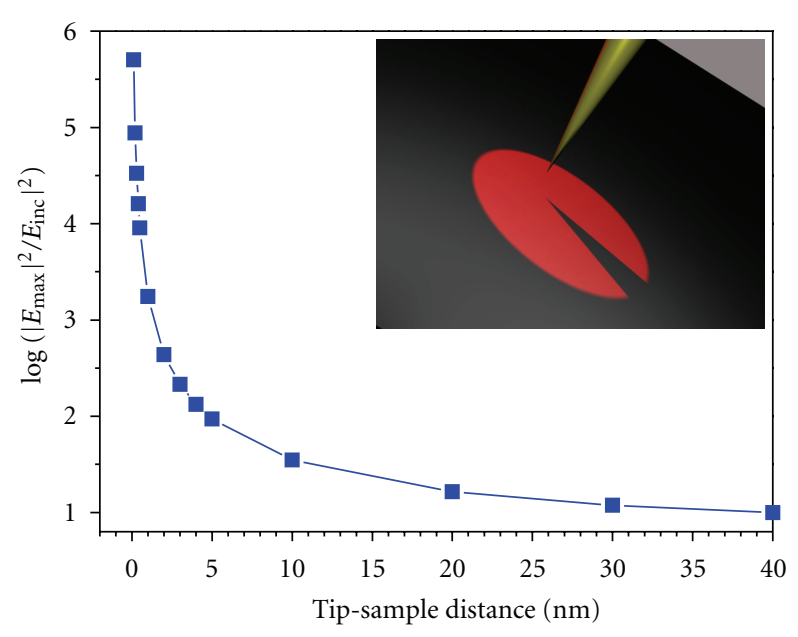

FIGURE 7: Raman enhancement factor estimated by Finite Elements Analysis (FEA) in the vicinity of the surface of interest, as a function of the tip-surface distance $(0.1$ to $40 \mathrm{~nm})$. The inset shows the tip approach from the incident beam and the shadowing of the incident beam during the approach process.

an estimation of the observed Raman enhancement factor $(G)$ below the tip apex. The contrast is defined as the ratio between the intensities of near-field and far-field signals $[53,54]$

$$
G=\frac{I_{\mathrm{NF}}}{I_{\mathrm{FF}}}=\text { contrast } \times \frac{\sigma_{\mathrm{tot}}}{\sigma_{\mathrm{NF}}}-1
$$

where $\sigma_{\mathrm{tot}}, \sigma_{\mathrm{NF}}, I_{\mathrm{FF}}$, and $I_{\mathrm{NF}}$ are respectively, the total section of analysis, the area of the near-field contribution (below the tip), the Raman intensity without any tip (far-field signal resulting from an area equal to $\sigma_{\mathrm{NF}}$ and the Raman intensity due to the tip (near-field signal resulting from an area equal to $\sigma_{\mathrm{NF}}$ ).

Applying this equation, the Raman enhancement factor below the tip apex is around $3.2 \times 10^{4}$. The quantitative estimation is in good agreement with already published papers $[28,29,54]$.

The second phase of these experiments starts when the tip is brought very close to the sample, less than $2 \mathrm{~nm}$. In this phase, tip displacements are controlled by the nanopositioning system. In order to manage the distance between the tip and the sample, the operator adjusts the setpoint value in which a feedback control is assigned. The contact between the tip and the sample occurs when the setpoint is equal to 0. Contrarily to FEM prediction (Figure 7), the Raman intensity enhancement slowly decreased when the tip is very close to SWNTC.

Comparison with modelling predicts that the peak will be between 0.3 and $0.5 \mathrm{~nm}$ from the surface. Let us remark that FEM calculations do not take into account the area of the sample hidden by the tip itself. An illustration of this possible phenomenon is showed in the inset of Figure 7. Regarding TERS experiments, we are assuming that this shadowing effect must be taken in consideration when the tip-sample distance is very small. This effect could probably be limited by reducing the dimensions of the silica tip apex and its aperture angle.

\section{Conclusion}

A side-illumination TERS device created by combining a nanopositioning system of a tip with a Raman spectrometer was described. The Raman signal is enhanced by the interaction of a nanoscale gold nanoparticle grafted onto sharp silica tips. This area was estimated to be $20 \mathrm{~nm}^{2}$ using finite element analysis. Even if a shadowing effect was clearly shown very close to the sample $(<2 \mathrm{~nm})$, our TERS experiments lead to an enhancement factor of about $3.2 \times 10^{4}$ of the Raman scattering intensity. The precision of the nanopositioning system and the high spatial resolution provided by the sharp tip are sufficient to scan and characterize non-transparent nanoobjects with nanometric spatial resolution. These preliminary experiments reveal the potential of the TERS device. Indeed, the benefits of a triple confocal Raman spectrometer, such as polarization control, choice of excitation wavelength (argon, krypton ionized, and solid semiconducting crystal lasers covering all the visible range), and high resolution are preserved by our setup. The possibility to explore low frequency bands of SWCNT by TipEnhanced Raman Spectroscopy opens up very interesting and promising developments of this technique.

\section{References}

[1] M. G. Albrecht and J. A. Creighton, "Anomalously intense Raman spectra of pyridine at a silver electrode," Journal of the American Chemical Society, vol. 99, no. 15, pp. 5215-5217, 1977.

[2] M. Fleischmann, P. J. Hendra, and A. J. McQuillan, "Raman spectra of pyridine adsorbed at a silver electrode," Chemical Physics Letters, vol. 26, no. 2, pp. 163-166, 1974.

[3] D. L. Jeanmaire and R. P. Van Duyne, "Surface Raman spectroelectrochemistry-part I. Heterocyclic, aromatic, and aliphatic amines adsorbed on the anodized silver electrode," Journal of Electroanalytical Chemistry, vol. 84, no. 1, pp. 1-20, 1977.

[4] K. Kneipp, Y. Wang, H. Kneipp et al., "Single molecule detection using surface-enhanced Raman scattering (SERS)," Physical Review Letters, vol. 78, no. 9, pp. 1667-1670, 1997.

[5] S. Nie and S. R. Emory, "Probing single molecules and single nanoparticles by surface-enhanced Raman scattering," Science, vol. 275, no. 5303, pp. 1102-1106, 1997.

[6] Z.-Y. Li and Y. Xia, "Metal nanoparticles with gain toward single-molecule detection by surface-enhanced Raman scattering," Nano Letters, vol. 10, no. 1, pp. 243-249, 2010.

[7] S. Rao, S. Raj, S. Balint et al., "Single DNA molecule detection in an optical trap using surface-enhanced Raman scattering," Applied Physics Letters, vol. 96, no. 21, Article ID 213701, 2010.

[8] J. A. Creighton, C. G. Blatchford, and M. G. Albrecht, "Plasma resonance enhancement of Raman scattering by pyridine adsorbed on silver or gold sol particles of size comparable to the excitation wavelength," Journal of the Chemical Society, Faraday Transactions 2, vol. 75, pp. 790-798, 1979.

[9] C. J. Orendorff, L. Gearheart, N. R. Jana, and C. J. Murphy, "Aspect ratio dependence on surface enhanced Raman 
scattering using silver and gold nanorod substrates," Physical Chemistry Chemical Physics, vol. 8, no. 1, pp. 165-170, 2006.

[10] K. Faulds, R. E. Littleford, D. Graham, G. Dent, and W. E. Smith, "Comparison of surface-enhanced resonance Raman scattering from unaggregated and aggregated nanoparticles," Analytical Chemistry, vol. 76, no. 3, pp. 592-598, 2004.

[11] T. Makiabadi, A. Bouvrée, V. Le Nader, H. Terrisse, and G. Louarn, "Preparation, optimization, and characterization of SERS sensor substrates based on two-dimensional structures of gold colloid," Plasmonics, vol. 5, no. 1, pp. 21-29, 2010.

[12] J. T. Bahns, F. Yan, D. Qiu, R. Wang, and L. Chen, "Holeenhanced raman scattering," Applied Spectroscopy, vol. 60, no. 9, pp. 989-993, 2006.

[13] L. Novotny and B. Hecht, Principles of Nano-Optics, Cambridge University Press, Cambridge, UK, 2006.

[14] J. Wessel, "Surface-enhanced optical microscopy," The Journal of the Optical Society of America, vol. 2, pp. 1538-1541, 1985.

[15] S. L. Sharp, R. J. Warmack, J. P. Goudonnet, I. Lee, and T. L. Ferrell, "Spectroscopy and imaging using the photon scanning-tunneling microscope," Accounts of Chemical Research, vol. 26, no. 7, pp. 377-382, 1993.

[16] A. Hartschuh, M. R. Beversluis, A. Bouhelier, and L. Novotny, "Tip-enhanced optical spectroscopy," Philosophical Transactions of the Royal Society A, vol. 362, no. 1817, pp. 807-819, 2004.

[17] B. Pettinger, "Tip-Enhanced Raman Spectroscopy (TERS)," Topics in Applied Physics, vol. 103, pp. 217-240, 2006.

[18] E. Bailo and V. Deckert, "Tip-enhanced Raman scattering," Chemical Society Reviews, vol. 37, no. 5, pp. 921-930, 2008.

[19] B. S. Yeo, J. Stadler, T. Schmid, R. Zenobi, and W. Zhang, "Tip-enhanced Raman spectroscopy-its status, challenges and future directions," Chemical Physics Letters, vol. 472, no. 1-3, pp. 1-13, 2009.

[20] R. Aroca, Surface-Enhanced Vibrational Spectroscopy, John Wiley and Sons, Chichester, UK, 2007.

[21] C. Vannier, B. S. Yeo, J. Melanson, and R. Zenobi, "Multifunctional microscope for far-field and tip-enhanced Raman spectroscopy," Review of Scientific Instruments, vol. 77, no. 2, article 023104, 2006.

[22] W. Zhang, B. S. Yeo, T. Schmid, and R. Zenobi, "Single molecule tip-enhanced Raman spectroscopy with silver tips," Journal of Physical Chemistry C, vol. 111, no. 4, pp. 1733-1738, 2007.

[23] E. Bailo and V. Deckert, "Tip-enhanced Raman spectroscopy of single RNA strands: towards a novel direct-sequencing method," Angewandte Chemie, vol. 47, no. 9, pp. 1658-1661, 2008.

[24] Y. Saito, P. Verma, K. Masui, Y. Inouye, and S. Kawata, "Nanoscale analysis of graphene layers by tip-enhanced near-field Raman spectroscopy," Journal of Raman Spectroscopy, vol. 40, no. 10, pp. 1434-1440, 2009.

[25] B. S. Yeo, E. Amstad, T. Schmid, J. Stadler, and R. Zenobi, "Nanoscale probing of a polymer-blend thin film with Tipenhanced Raman spectroscopy," Small, vol. 5, no. 8, pp. 952960, 2009.

[26] L. G. Cançado, A. Hartschuh, and L. Novotny, "Tip-enhanced Raman spectroscopy of carbon nanotubes," Journal of Raman Spectroscopy, vol. 40, no. 10, pp. 1420-1426, 2009.

[27] D. Mehtani, N. Lee, R. D. Hartschuh et al., "Nano-Raman spectroscopy with side-illumination optics," Journal of Raman Spectroscopy, vol. 36, no. 11, pp. 1068-1075, 2005.
[28] Y. Saito, M. Motohashi, N. Hayazawa, M. Iyoki, and S. Kawata, "Nanoscale characterization of strained silicon by tipenhanced Raman spectroscope in reflection mode," Applied Physics Letters, vol. 88, no. 14, Article ID 143109, 2006.

[29] N. Lee, R. D. Hartschuh, D. Mehtani et al., "High contrast scanning nano-Raman spectroscopy of silicon," Journal of Raman Spectroscopy, vol. 38, no. 6, Article ID 143109, pp. 789796, 2007.

[30] G. Picardi, Q. Nguyen, J. Schreiber, and R. Ossikovski, "Comparative study of atomic force mode and tunneling mode tip-enhanced Raman spectroscopy," The European Physical Journal, vol. 40, no. 2, pp. 197-201, 2007.

[31] P. G. Gucciardi, M. Lopes, R. Déturche, C. Julien, D. Barchiesi, and M. Lamy De La Chapelle, "Light depolarization induced by metallic tips in apertureless near-field optical microscopy and tip-enhanced Raman spectroscopy," Nanotechnology, vol. 19, no. 21, Article ID 215702, 2008.

[32] M. Motohashi, N. Hayazawa, A. Tarun, and S. Kawata, "Depolarization effect in reflection-mode tip-enhanced Raman scattering for Raman active crystals," Journal of Applied Physics, vol. 103, no. 3, Article ID 034309, 2008.

[33] A. Merlen, J. C. Valmalette, P. G. Gucciardi, M. Lamy de la Chapelle, A. Frigout, and R. Ossikovski, "Depolarization effects in tip-enhanced raman spectroscopy," Journal of Raman Spectroscopy, vol. 40, no. 10, pp. 1361-1370, 2009.

[34] B. Ren, G. Picardi, and B. Pettinger, "Preparation of gold tips suitable for tip-enhanced Raman spectroscopy and light emission by electrochemical etching," Review of Scientific Instruments, vol. 75, no. 4, pp. 837-841, 2004.

[35] C. C. Neacsu, S. Berweger, and M. B. Raschke, “Tip-enhanced raman imaging and nanospectroscopy: Sensitivity, symmetry, and selection rules," Nanobiotechnology, vol. 3, no. 3-4, pp. 172-196, 2007.

[36] C. Williams and D. Roy, "Fabrication of gold tips suitable for tip-enhanced Raman spectroscopy," Journal of Vacuum Science and Technology B: Microelectronics and Nanometer Structures, vol. 26, no. 5, pp. 1761-1764, 2008.

[37] R. M. Stöckle, Y. D. Suh, V. Deckert, and R. Zenobi, "Nanoscale chemical analysis by tip-enhanced Raman spectroscopy," Chemical Physics Letters, vol. 318, no. 1-3, pp. 131-136, 2000.

[38] M. Chaigneau, G. Ollivier, T. Minea, and G. Louarn, "Nanoprobes for near-field optical microscopy manufactured by substitute-sheath etching and hollow cathode sputtering," Review of Scientific Instruments, vol. 77, no. 10, Article ID 103702, 2006.

[39] R. M. Stöckle, Y. D. Suh, V. Deckert, and R. Zenobi, "Nanoscale chemical analysis by tip-enhanced Raman spectroscopy," Chemical Physics Letters, vol. 318, no. 1-3, pp. 131-136, 2000.

[40] S. S. Kharintsev, G. G. Hoffmann, P. S. Dorozhkin, G. De With, and J. Loos, "Atomic force and shear force based tip-enhanced Raman spectroscopy and imaging," Nanotechnology, vol. 18, no. 31, Article ID 315502, 2007.

[41] N. Anderson, A. Hartschuh, and L. Novotny, "Near-field Raman microscopy," Materials Today, vol. 8, no. 5, pp. 50-54, 2005.

[42] A. L. Demming, F. Festy, and D. Richards, "Plasmon resonances on metal tips: Understanding tip-enhanced Raman scattering," Journal of Chemical Physics, vol. 122, no. 18, Article ID 184716, pp. 1-7, 2005.

[43] N. Hayazawa, Y. Saito, and S. Kawata, "Detection and characterization of longitudinal field for tip-enhanced Raman spectroscopy," Applied Physics Letters, vol. 85, no. 25, pp. 6239$6241,2004$. 
[44] K. F. Domke and B. Pettinger, “Tip-enhanced Raman spectroscopy of $6 \mathrm{H}-\mathrm{SiC}$ with graphene adlayers: selective suppression of E1 modes," Journal of Raman Spectroscopy, vol. 40, no. 10, pp. 1427-1433, 2009.

[45] W. X. Sun and Z. X. Shen, "Apertureless near-field scanning Raman microscopy using reflection scattering geometry," Ultramicroscopy, vol. 94, no. 3-4, pp. 237-244, 2003.

[46] D. Roy, J. Wang, and M. E. Welland, "Nanoscale imaging of carbon nanotubes using tip enhanced Raman spectroscopy in reflection mode," Faraday Discussions, vol. 132, pp. 215-225, 2006.

[47] R. J. C. Brown, J. Wang, R. Tantra, R. E. Yardley, and M. J. T. Milton, "Electromagnetic modelling of Raman enhancement from nanoscale substrates: A route to estimation of the magnitude of the chemical enhancement mechanism in SERS," Faraday Discussions, vol. 132, pp. 201-213, 2006.

[48] A. Jorio, M. A. Pimenta, A. G. Souza Filho, R. Saito, G. Dresselhaus, and M. S. Dresselhaus, "Characterizing carbon nanotube samples with resonance Raman scattering," New Journal of Physics, vol. 5, pp. 139.1-139.17, 2003.

[49] N. Anderson, A. Hartschuh, S. Cronin, and L. Novotny, "Nanoscale vibrational analysis of single-walled carbon nanotubes," Journal of the American Chemical Society, vol. 127, no. 8, pp. 2533-2537, 2005.

[50] A. Hartschuh, H. Qian, A. J. Meixner, N. Anderson, and L. Novotny, "Nanoscale optical imaging of excitons in singlewalled carbon nanotubes," Nano Letters, vol. 5, no. 11, pp. 2310-2313, 2005.

[51] N. Anderson, A. Hartschuh, and L. Novotny, "Chirality changes in carbon nanotubes studied with near-field Raman spectroscopy," Nano Letters, vol. 7, no. 3, pp. 577-582, 2007.

[52] T. A. Yano, T. Ichimura, A. Taguchi et al., "Confinement of enhanced field investigated by tip-sample gap regulation in tapping-mode tip-enhanced Raman microscopy," Applied Physics Letters, vol. 91, no. 12, Article ID 121101, 2007.

[53] T. Schmid, A. Messmer, B. S. Yeo, W. Zhang, and R. Zenobi, "Towards chemical analysis of nanostructures in biofilms II: tip-enhanced Raman spectroscopy of alginates," Analytical and Bioanalytical Chemistry, vol. 391, no. 5, pp. 1907-1916, 2008.

[54] A. Tarun, N. Hayazawa, M. Motohashi, and S. Kawata, "Highly efficient tip-enhanced Raman spectroscopy and microscopy of strained silicon," Review of Scientific Instruments, vol. 79, no. 1, Article ID 013706, 2008. 

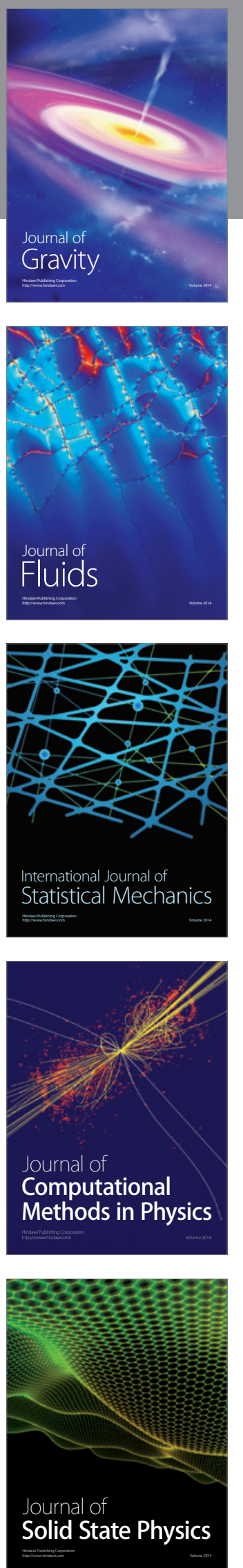

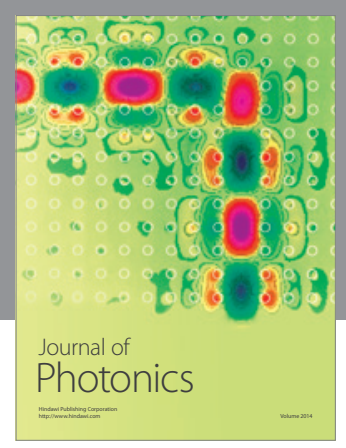

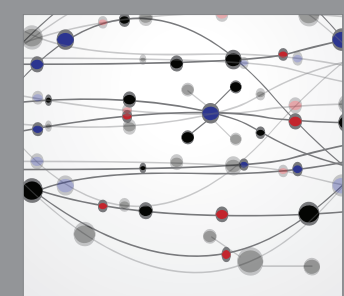

The Scientific World Journal
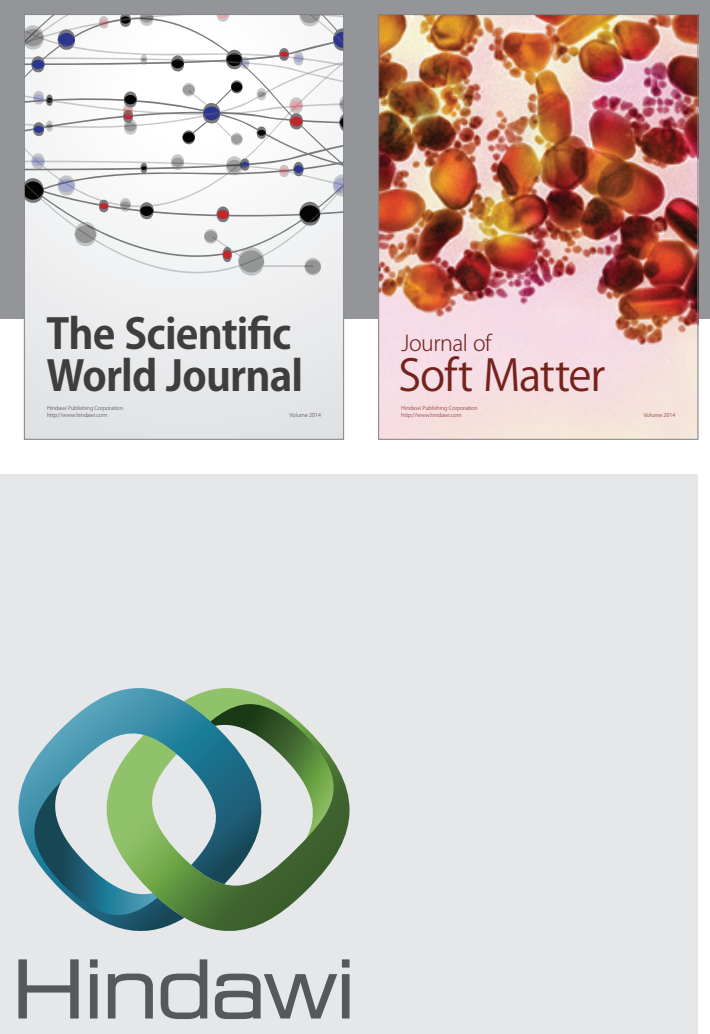

Submit your manuscripts at

http://www.hindawi.com
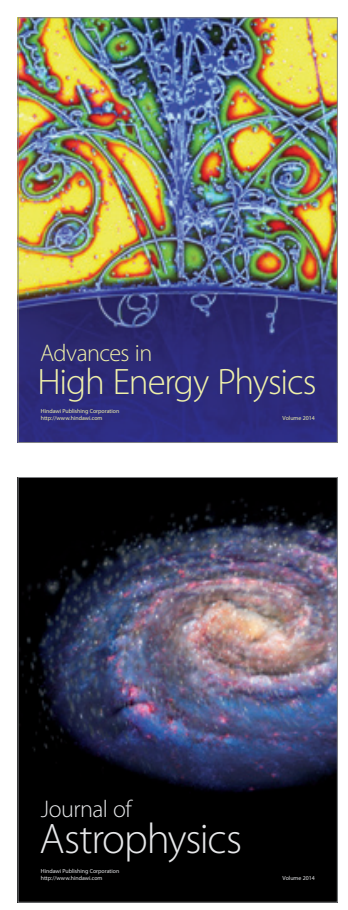
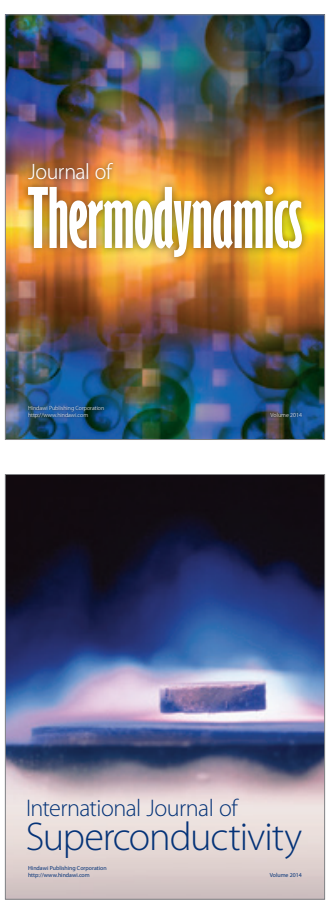
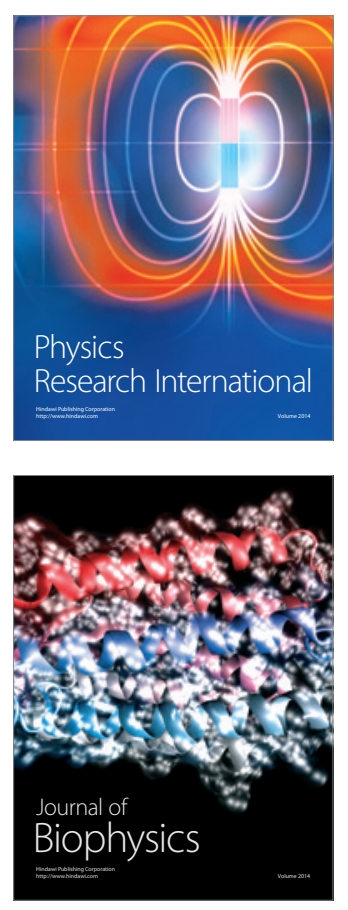
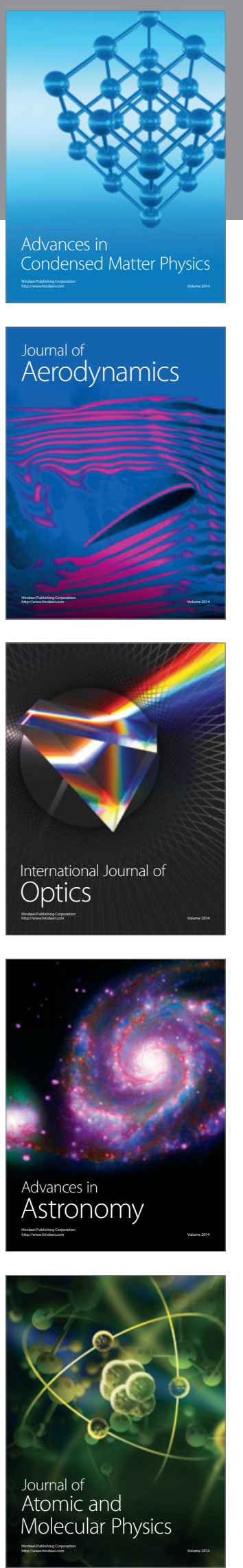